\title{
VERIFICATION OF DIAGNOSTIC ACCURACY OF CONE BEAM COMPUTED TOMOGRAPHIC IMAGING MODES FOR DETECTION OF VERTICAL ROOT FRACTURES
}

\author{
Iman Dakhli * and Omniya Abu El-Dahab*
}

\begin{abstract}
Aim: To assess the diagnostic performance of CBCT for enhancing the detection of vertical fractures with non-displacing root fragments in non-filled teeth to establish their presence/absence with different voxel sizes.

Methods: The study material comprised 45 extracted single and birooted, unrestored human mandibular and maxillary premolars teeth. In 30 of the teeth, VRFs were created in the buccolingual plane after root canal accesses were made. The remainders of the roots (15) were kept intact. The teeth were examined with CBCT (i-Cat, Imaging Sciences Int., Hatfield, PA) at the original voxel size of $125 \mu \mathrm{m}$. These raw images were then down sampled to achieve voxel sizes of 200 , and $250 \mu \mathrm{m}$.
\end{abstract}

Results: It was observed that the highest diagnostic accuracy (100.0\%) was found with voxel size 0.125 in Groups I and II as well as voxel size 0.200 in Group II. The lowest diagnostic accuracy (75.0\%) was found with voxel size 0.250 in Group III.

Conclusion: We think that not only the voxel size exerts little influence on the detection of root fractures but also the accuracy levels were lower when using the voxels of greater thickness.

\section{INTRODUCTION}

Vertical root fracture (VRF) is defined as a root fracture in the longitudinal direction, extending from the root to the crown on facial or lingual surfaces. Both endodontically treated teeth and non-endodontically treated teeth can be involved. Most VRFs occur in endodontically treated teeth, and the symptoms are similar to those of chronic apical periodontitis or chronic periodontitis. It has been hypothesized that the reasons may be either oversized root canal therapy that weakens the canal's dentin walls or the presence of an endodontic post. Masticatory forces lead to a higher prevalence of longitudinal fractures in treated teeth compared with untreated teeth. VRF is usually iatrogenic and can occur after the insertion of retention screws or

* Lecturer of Oral and Maxillofacial Radiology, Faculty of Oral and Dental Medicine, Cairo University, Egypt. 
pins. Endodontically treated uncrowned posterior teeth or inappropriate tooth selection as bridge abutments are most at risk. ${ }^{(1-7)}$

Correct diagnosis of vertical root fractures is a challenge for dental surgeons, and the early detection of these fractures would directly influence treatment planning. The diagnostic process should include a thorough analysis of the case history and a detailed clinical examination, as well as an evaluation of the bone and tooth structure. Therefore, it is essential to order complementary exams. VRF lacks specific radiographic signs and symptoms, thus making a precise diagnosis of VRF in endodontically treated teeth difficult. Low prevalence of VRFs estimated previously could be underestimated. Many cases of VRFs could not be definitively diagnosed until tooth extraction. ${ }^{(4,7-11)}$

Early diagnosis of VRF is imperative to avoid unnecessary invasive surgery and/or extraction of the tooth. The clinical presentation and radiographic appearance of a vertical root fracture (VRF) frequently pose a diagnostic dilemma and a true challenge to the clinician because the related clinical and radiographic signs are not pathognomonic. ${ }^{(12,13)}$

Although clinical and radiographic features may not definitively identify a fractured root, clinicians often base their diagnosis on the patient's clinical signs and symptoms, or on direct and indirect features identified on conventional radiographs. The presence of a radiolucent "line" between displaced tooth root fragments is a direct radiographic feature of a root fracture. In contrast, radiographic features such as localized widening of the periodontal ligament space, and the "halo" appearance, which can be described as either "combined periapical and perilateral radiolucency along the side of the root, lateral periodontal radiolucency along the side of the root, or angular radiolucency from the crestal bone terminating along the root side," is often found in the presence of VRFs occur in molars, radiolucency can be found in the furcation area is indirect sign of a fracture. ${ }^{(6,14)}$
The diagnosis of some root fractures on conventional radiographs may be complicated and they can be overlooked if the x-ray beam does not pass along the fracture line, also because of the lack of specific clinical signs and symptoms. CBCT has been suggested as the imaging modality of choice for detecting VRF. ${ }^{(3,4,7,12-16)}$

Cone beam $\mathrm{CT}$ (CBCT) is a relatively new and alternative imaging modality in the maxillofacial region that has gained broad acceptance in the dental profession. CBCT has recently received attention as a new standard diagnostic tool because it is able to accurately represent the $3 \mathrm{D}$ shape and position of the jaw and provides high-resolution multiplanar images, thus, improves the diagnostic capacity in dentistry for general practice. . As an emerging technology, the CBCT has distinct advantages of being a low-cost and dose-saving imaging modality, creating thereby a promising alternative to conventional radiography and even to the highend medical CT scanners. With the advantages of low cost, easy accessibility, and low radiation dose compared with multislice computed tomography. However, the major obstacle is that $\mathrm{CBCT}$ imaging cannot provide detailed surface dental morphology and accurate interocclusal relationships, owing to the limited scanning resolution and streak artifacts caused by radiopaque dental restorations (e.g., metal crown) or orthodontic brackets. ${ }^{(3,8,17-23)}$

Digital images are composed of elements called pixels. A pixel is a picture element, a square in a 2D matrix. Each pixel has a specific size, intensity value, and location within the matrix. A voxel is a volume element, and it is the cubed form of a pixel, having a third dimension that represents the density value of a point in 3D space, which is analogous to a pixel. Because the CBCT data were composed of many voxels, each with its own gray value according to the radiation absorbed, the computer used the voxels to reconstruct the 3D model. Threedimensional images are composed of voxels instead 
of the pixels in 2D digital images. The size of each voxel is determined by its height, width, and thickness, and a voxel is the smallest element of the $3 \mathrm{D}$ radiograph image volume. CBCT images use isotropic voxels, meaning they have the same height, width and thickness. The isotropic nature of the voxels affords the same quality as the original image in reconstructions. The displayed voxel size is a direct product of the native pixel dimensions. This technique results in isotropic voxels. Voxel sizes in CBCT imaging range from $0.076 \mathrm{~mm}$ to 0.4 $\mathrm{mm}$, depending on the particular unit and protocol being used. ${ }^{(16,20,24)}$

\section{Methodology}

The study material comprised 45 extracted single and birooted, unrestored (except 2 with amalgam fillings) human mandibular and maxillary premolars teeth out of 60 teeth that were collected and randomly selected and inspected with a magnifying lens to confirm the absence of defects or root fractures and 15 of those 60 teeth were ruined during the attempts to induce the vertical root fracture (VRFs). Individual tooth surfaces were hand scaled to remove any remaining soft tissue. All teeth were stored in distilled water at $-20^{\circ} \mathrm{C}$. In 30 of the teeth, VRFs were created in the buccolingual plane after root canal accesses were made in those 30 teeth either by gently tapping with a hammer over a screw-type root canal pin inserted into the tooth using controlled pressure or by rotation of the root canal pin in anti-clock-wise direction while the tooth was placed in an acrylic mould act as soft foundation base. After total separation of the fragments, they were placed together, returning to their original position, to simulate the immediate post-trauma situation in which no edema or granulation tissue has displaced the fragments yet.

To be able to clearly identify the fracture site, the fracture line was delineated on the root with a blue pen after gluing. The remainders of the roots ${ }^{(15)}$ were kept intact.
The roots were uniformly covered with a layer of utility wax (Cavex Holland BV, Netherland) to simulate the radiographic aspect of periodontal space and the alveolar cortical bone. The remaining 15 intact teeth served as control samples. Teeth were randomly distributed into 3 groups, numbered, and embedded in dental stone in a straight line. The teeth were examined with CBCT (i-Cat, Imaging Sciences Int., Hatfield, PA) at the original voxel size of $125 \mu \mathrm{m}$. These raw images were then down sampled to achieve voxel sizes of 200 , and $250 \mu \mathrm{m}$. One Scan was performed for each tooth. The set-up was as follows: $120 \mathrm{kVp}, 5 \mathrm{~mA}$ and 7 seconds. Images were obtained by volume data of cone type with a field size of (45-48) $\mathrm{mm}$.

\section{Data Analysis}

The axial, coronal, and sagittal tomographic slices of the data sets were created in image analysis software (i-CAT vision software, version 1.7.0.7). Two blinded and calibrated experienced oral-maxillofacial radiologists with at least 10 years of experience assessed the images on each slice orientation independently of the dataset with different voxel sizes. Viewing was performed under uniform subdued lighting in a quiet and secluded room. Scrolling axial, sagittal, and coronal slices interactively, searching for a radiolucent thin line along the root using the imaging systems' own software and enhancement tools at random order. Each observer judged whether or not a fracture was present in each dataset, i.e., regardless of in which sectional plane it was seen (yes/no in each root). The calibration included training on the radiographic features of VRF on CBCT scans. The visibility of a radiolucent fracture line crossing the root either completely or partially on at least two consecutive slices was the main radiographic feature for detecting a VRF. Each observer scored the presence of VRF on a 3-point rank scale:

0 : absent

1: present, yet poorly defined

2: Present and well defined 
The root was considered fractured when a fracture line was detected on any one of the three slices. Each observer evaluated the 3 voxel resolutions $(0.125,0.2,0.25 \mathrm{~mm})$ by using axial, sagittal, and coronal directions, and a single score was obtained for each tooth. And at a 1-week interval, the same observations were repeated. The responses of the 2 radiologists were compared by the gold standard, and conflicting data were reassessed 2 weeks after the first evaluation by the same examiners working together, with the aim of reaching a consensus to obtain the final responses (Fig. 1).

Images were compared with the gold standard using receiver operating characteristic (ROC) analysis to evaluate observer ability in differentiating between teeth with and without VRF. Each reading of each observer was evaluated independently to determine differences in diagnostic accuracy among image types.

Fracture/non-fracture recordings were categorized as follows: correct identification of a nonfractured root (true negative); correct identification of fracture site in a fractured root (true positive); identification of a fracture in a non-fractured root (false positive); no identification of a fracture in a fractured root (false negative) and incorrect identification of the fracture site in a fractured root (false negative).

\section{Statistical Analysis}

Inter observer agreement (reliability) was assessed using Kappa statistic. Kappa statistic values are interpreted as follows; $0-0.2$ : weak agreement, $0.2-0.4$ : fair agreement, $0.4-0.6$ : moderate agreement, $0.6-0.8$ : good agreement, $0.8-0.99$ : very good agreement while a value of 1 indicates perfect agreement.

ROC (Receiver Operating Characteristic) curve was constructed to evaluate diagnostic accuracy
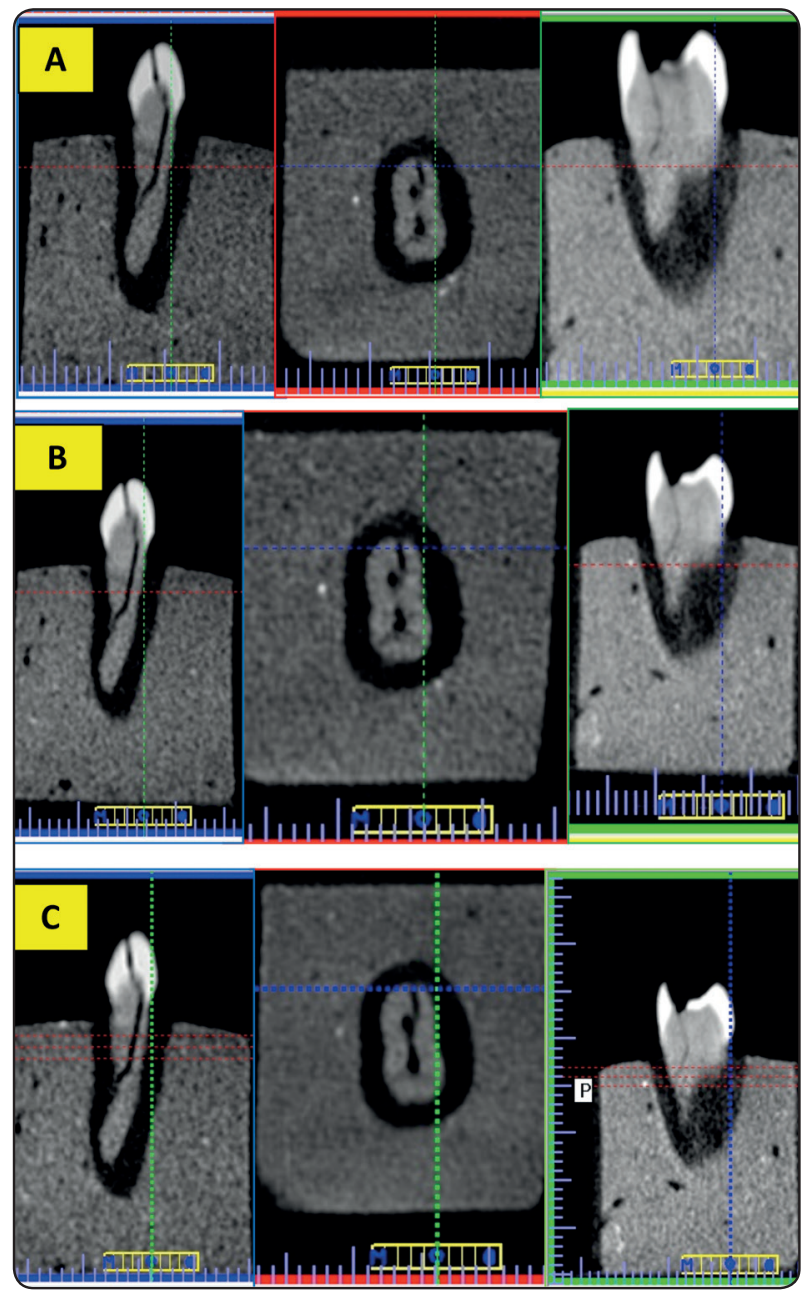

Fig. (1) i-CAT CBCT reconstructions images of a fractured root with 3 different voxels in 3 planes. (A) $0.125-\mathrm{mm}$ voxel; Axial plane (red color coded) sagittal plane(green color code) and coronal plane (blue color coded), (B) 0.200-mm voxel; Axial plane(red dotted line) sagittal plane(green color coded) and coronal plane (blue color coded), (C) 0.250-mm voxel; Axial plane(red color code) sagittal plane(green color coded) and coronal plane (blue color coded)

measures of different voxel sizes in the three groups. Areas under the ROC curve (AUCs) of the different voxel sizes were compared using $z$-statistic. For ROC curve analysis; Score 0 represents no fracture while Scores 1 and 2 were considered as presence of fracture. 
The significance level was set at $\mathrm{P} \leq 0.05$. Interobserver agreement analysis was performed with IBM $^{\circledast}$ SPSS $^{\circledast}$ Statistics Version 20 for Windows.

ROC curve analysis was performed with MedCalc Version 11.3 for Windows (MedCalc Software bvba).

\section{RESULTS}

\section{Inter-observer agreement (reliability)}

Results of Kappa statistic regarding interobserver agreement in the three groups is presented in (Table. 1).

In Group I; there was moderate to good interobserver agreement. voxel size 0.125 showed the highest agreement followed by voxel size 0.200 while voxel size 0.250 showed the lowest agreement.

As regards Group II; there was good to very good inter-observer agreement. voxel size 0.200 showed the highest agreement followed by voxel size 0.125 while voxel size 0.250 showed the lowest agreement.

While for Group III; there was moderate to good inter-observer agreement. voxel size 0.125 showed the highest agreement followed by voxel size 0.200 while voxel size 0.250 showed the lowest agreement.

Comparing the inter-observer agreement between the three groups revealed that for voxel sizes 0.125 and 0.200; Group II showed the highest agreement followed by Group III while Group I showed the lowest agreement. While for voxel size 0.250; Group II showed the highest agreement followed by Group I while Group III showed the lowest agreement.
TABLE (1) Results of Kappa statistic for interobserver agreement in the three groups

\begin{tabular}{|c|c|c|}
\hline Group & Voxel size & Kappa value \\
\hline \multirow{3}{*}{ Group I } & 0.125 & 0.619 \\
\cline { 2 - 3 } & 0.200 & 0.524 \\
\cline { 2 - 3 } & 0.250 & 0.494 \\
\hline \multirow{4}{*}{ Group II } & 0.125 & 0.805 \\
\cline { 2 - 3 } & 0.200 & 0.899 \\
\cline { 2 - 3 } & 0.250 & 0.621 \\
\hline \multirow{4}{*}{ Group III } & 0.125 & 0.750 \\
\cline { 2 - 3 } & 0.200 & 0.633 \\
\cline { 2 - 3 } & 0.250 & 0.484 \\
\hline
\end{tabular}

\section{Diagnostic accuracy (ROC curve analysis)}

Results of ROC curve analysis are presented in (Table. 2). It was observed that the highest diagnostic accuracy (100.0\%) was found with voxel size 0.125 in Groups I and II as well as voxel size 0.200 in Group II. The lowest diagnostic accuracy (75.0\%) was found with voxel size 0.250 in Group III.

\section{Comparison between diagnostic accuracy of dif- ferent Voxel sizes within each group}

Results of ROC curve analysis and pair-wise comparisons between different voxel sizes are presented in (Table. 3).

In Group I; the diagnostic accuracy with voxel sizes $0.125,0.200$ and 0.250 were $100.0 \%$, $87.5 \%$ and $81.2 \%$, respectively. However, pair-wise comparisons revealed that here was no statistically significant difference between the three voxel sizes.

As regards Group II; the diagnostic accuracy with voxel sizes $0.125,0.200$ and 0.250 were $100.0 \%, 100.0 \%$ and $93.8 \%$, respectively. However, pair-wise comparisons revealed that there was no statistically significant difference between the three voxel sizes.

\footnotetext{
${ }^{\circledR}$ IBM Corporation, NY, USA.

${ }^{\circledR}$ SPSS, Inc., an IBM Company.
} 
TABLE (2) Sensitivity, specificity, predictive values, diagnostic accuracy, Area Under the ROC curve (AUC), standard error (SE) and 95\% confidence interval (95\% CI) of the three groups with different voxel sizes

\begin{tabular}{|c|c|c|c|c|c|c|c|c|c|}
\hline \multirow{3}{*}{ Group } & Voxel size & Sensitivity $\%$ & $\begin{array}{c}\text { Specificity } \\
\%\end{array}$ & $\begin{array}{c}+\mathrm{PV} \\
\%\end{array}$ & $\begin{array}{c}-\mathrm{PV} \\
\%\end{array}$ & $\begin{array}{c}\text { Diagnostic } \\
\text { accuracy } \%\end{array}$ & AUC & SE & $95 \%$ CI \\
\hline \multirow{3}{*}{ Group I } & 0.125 & 100.0 & 100.0 & 100.0 & 100.0 & 100.0 & 1.000 & 0.000 & $0.794-1.000$ \\
\cline { 2 - 10 } & 0.200 & 81.8 & 100.0 & 100.0 & 71.4 & 87.5 & 0.909 & 0.061 & $0.660-0.994$ \\
\cline { 2 - 11 } & 0.250 & 72.7 & 100.0 & 100.0 & 62.5 & 81.2 & 0.864 & 0.070 & $0.603-0.981$ \\
\hline \multirow{3}{*}{ Group II } & 0.125 & 100.0 & 100.0 & 100.0 & 100.0 & 100.0 & 1.000 & 0.000 & $0.794-1.000$ \\
\cline { 2 - 10 } & 0.200 & 100.0 & 100.0 & 100.0 & 100.0 & 100.0 & 1.000 & 0.000 & $0.794-1.000$ \\
\hline \multirow{3}{*}{ Group III } & 0.1250 & 90.0 & 100.0 & 100.0 & 85.7 & 93.8 & 0.950 & 0.050 & $0.715-0.999$ \\
\cline { 2 - 10 } & 0.200 & 100.0 & 75.0 & 88.9 & 100.0 & 91.7 & 0.875 & 0.125 & $0.564-0.991$ \\
& 0.250 & 75.0 & 75.0 & 85.7 & 60.0 & 75.0 & 0.750 & 0.149 & $0.428-0.945$ \\
\hline
\end{tabular}

$+P V$ : Positive Predictive Value, -PV: Negative Predictive Value

TABLE (3) Results of z-test for pair-wise comparisons between the different voxel sizes within each group

\begin{tabular}{|c|c|c|c|}
\hline \multirow{3}{*}{ Group } & Voxel size comparisons & $z$ - statistic & $P$-value \\
\hline \multirow{3}{*}{ Group I } & 0.125 Vs. 0.200 & 1.491 & 0.136 \\
\cline { 2 - 4 } & 0.125 Vs. 0.250 & 1.936 & 0.053 \\
\cline { 2 - 4 } & 0.200 Vs. 0.250 & 1.000 & 0.317 \\
\hline \multirow{3}{*}{ Group II } & 0.125 Vs. 0.200 & 0.000 & 1.000 \\
\cline { 2 - 4 } & 0.125 Vs. 0.250 & 1.000 & 0.317 \\
\hline \multirow{3}{*}{ Group III } & 0.200 Vs. 0.250 & 1.000 & 0.317 \\
\cline { 2 - 4 } & 0.125 Vs. 0.200 & 0.000 & 1.000 \\
\cline { 2 - 4 } & 0.125 Vs. 0.250 & 1.528 & 0.127 \\
\hline
\end{tabular}

*: Significant at $P \leq 0.05$

While for Group III; the diagnostic accuracy with voxel sizes $0.125,0.200$ and 0.250 were $91.7 \%$, $91.7 \%$ and $75.0 \%$, respectively. However, pair-wise comparisons revealed that there was no statistically significant difference between the three voxel sizes.

\section{DISCUSSION}

In vitro studies performed under simulated conditions. In all of them, the presence of fractures was evaluated through direct visualization of the fracture as the presence of a radiolucent space among 2 or more tooth fragments. On the contrary, 
in clinical practice, the presence of a VRF could also be postulated indirectly, on the basis of the presence of certain radiographic features. The diagnosis of VRF is relatively limited and is composed mainly of in vitro studies that do not reflect the true clinical scenario. ${ }^{(6)}$

In this study, the roots were uniformly covered with a layer of utility wax (Cavex Holland BV, Netherland) to simulate the radiographic aspect of periodontal space and the alveolar cortical bone as close as possible to reality. However, in a number of cases, the diagnosis of root fractures is associated with other clinical radiographic findings such as alterations in the periodontal ligament space, osteolytic lesions, and pain while chewing. Such conditions are not amenable to simulation, constituting a limitation of in vitro studies.

It is known that an in vivo study would have been more realistic; however, the ethical aspects in research involving humans do not allow the performance of several x-ray exams with the same purpose of diagnosis. Therefore, conducting previous in vitro studies is necessary to allow the development of posterior clinical research. ${ }^{(2,3,11,15,25)}$

Using an ex vivo model and teeth with artificially induced VRFs; we focused our attention on the detection of the fracture line, which is not the sole component of a VRF. Therefore, we were unable to analyze radiologic indirect signs of bony lesions which can be seen as a "halo" lesion, perilateral radiolucency and angular resorption of the crestal bone, combined with diffuse or defined, but not corticated borders. Fragments were fixed tightly together and relocated in their original position. This made the appearance of the fracture lines similar to that in immediate post-trauma cases and thus difficult to detect.

The diagnosis of VRF is usually problematic because it often necessitates prediction rather than a definite identification. Because radiographs and CBCT scans acquired by using different imaging parameters cannot exactly visualize these fractures, the location and defect size cannot always be objectively assessed until the tooth has been extracted or a simultaneous flap operation has been performed for direct visualization. ${ }^{(16)}$

From a practical standpoint, the detection of vertical root fractures with non-displacing fragments is frequently difficult, so early clinical management is often based on a suspicion rather than definitive diagnosis. Even in CBCT scans of some suspected teeth, fracture lines may not be visualized. $(4,10,15)$

In our study, the observers independently assessed the images twice. This procedure is more objective. It would be helpful if future studies reported the validity as well as the reliability of a new diagnostic tool such as the CBCT. In this way the benefits to the patient of each exposure can outweigh any risks.

To calculate validity, a gold standard is needed. Although findings after tooth extraction are probably the nearest as we can come to a real gold standard, because extraction allows the complete root surfaces of a tooth to be inspected, however, because extraction is not always feasible in a clinical situation, the gold standard is slightly compromised. ${ }^{(13)}$

The term CBCT does not refer to a single imaging protocol. There are several parameters that influence the quality of CBCT images, including $\mathrm{X}$-ray beam factors, the size of the FOV, the detector type, and the size of the reconstructed voxels. These parameters vary between CBCT units and with different imaging protocols of the same CBCT unit. Thus, when designing CBCT imaging protocols, it is important to adjust these parameters to produce images that are optimal for a specific diagnostic task. ${ }^{(26)}$

Image quality has been described as the visibility of diagnostically important structures in the CT image. Voxel size has been reported to have a positive correlation with image quality (contrast and resolution) and exposure dose. It is known 
that with smaller voxel sizes, radiation exposure would be higher. Without sacrificing image quality and adopting the ALARA "as low as reasonably achievable" principle, the ability to select various voxel settings would be helpful in reducing the radiation dose to the patient. ${ }^{(15,16,27)}$

Voxel size is directly linked to the spatial resolution of an image. In general, the smaller the voxel size, the better both resolution and details. We think that the difference in size of the voxels tested might not have been sufficient to increase the spatial resolution of the images to any significant degree. Furthermore, other studies have shown that voxel size exerts little influence on the detection of external root resorption, internal root resorption, occlusal caries, root fractures, or preoperative implant measurements, the accuracy levels were lower when using the voxel of greater thickness. The second factor affecting image quality is the type of CBCT detector used; FPD detectors were shown to produce better quality images than IIT/CCD detectors. The third factor affecting image quality is image reconstruction. Therefore, Proper voxel selection for diagnosis of VRF is important. ${ }^{(2,3,5,7,10,11,15,16,18,25,26,28)}$

CBCT scans provide reliable information for diagnosing VRFs in non-endodontically treated teeth. Variation of the acquisition protocols in performing the exam by using voxels of different sizes is an alternative that can improve image quality. ${ }^{(4,11,15,16)}$

One advantage of this study design is the ability to isolate the test variable, which is the voxel size, while maintaining consistency in all other variables through the manipulation of a single CBCT acquisition into three different viewing parameters, This will eliminate the potential confounding factors from the data analysis, thereby permitting stronger and more focused study conclusions.

Ex vivo studies support the use of CBCT scans for the detection of VRFs in endodontically treated teeth. However, the VRFs in these studies were artificially created, which could be different from the "naturally" occurring ones. Also, when the detection of VRFs by different CBCT scanners was compared, there was always a difference in their detection accuracy both in ex vivo and in vivo. ${ }^{(3,9}$. $12,13,15,16,29)$

In the current study Group I; showed moderate to good inter-observer agreement. As regards Group II; there was good to very good inter-observer agreement. While for Group III; there was moderate to good inter-observer agreement. Comparing the inter-observer agreement between the three groups revealed that for voxel sizes 0.125 and 0.200 ; Group II showed the highest agreement followed by Group III while Group I showed the lowest agreement. While for voxel size 0.250; Group II showed the highest agreement followed by Group I while Group III showed the lowest agreement.

Hassan et al $2009{ }^{(9)}$ reported that the overall agreement among the observers was moderate $(\mathrm{k}$ $=0.521)$. There was no significant difference in overall accuracy among the observers for detecting VRFs by both CBCT scans and PRs $(\mathrm{p}=0.76)$. While according to Hassan et al $2010{ }^{(15)}$ the overall agreement between the observers was fair $(\mathrm{k}=$ $0.385)$. The agreement for the $\mathrm{i}-\mathrm{CAT}$ was good $(\mathrm{k}=$ 0.68 ), and it was better when compared with those for other systems.

In the current study results of ROC curve analysis were showed that the highest diagnostic accuracy $(100.0 \%)$ was found with voxel size 0.125 in Groups I and II as well as voxel size 0.200 in Group II. The lowest diagnostic accuracy (75.0\%) was found with voxel size 0.250 in Group III. However, pair-wise comparisons revealed that there was no statistically significant difference between the three voxel sizes in all groups.

According to Corbella et al $2014^{(6)}$ In vitro studies on unfilled teeth found high $(>0.90)$ specificity for CBCT when voxel size was less than $0.3 \mathrm{~mm}$, whereas sensitivity was less than 0.90 when voxel size was less than $0.25 \mathrm{~mm}$. The results 
indicated that compared with periapical radiographs, CBCT had similar specificity (the ability to detect all true negatives) but higher sensitivity (the ability to detect true positives) markedly lower sensitivity than CBCT when voxel size was less than $0.25 \mathrm{~mm}$.

Damstra et al $2010{ }^{(30)}$ justify the use of CBCT-derived surface models for orthodontic and craniofacial treatment planning. There was no difference between the CBCT measurements of the $0.4-\mathrm{mm}$ and $0.25-\mathrm{mm}$ voxel resolution groups compared with anatomic truth. These results confirm the results of Ballrick et al $2008{ }^{(31)}$ and suggest that $0.4-\mathrm{mm}$ voxel resolution is adequate for measurement of craniofacial structures.

Confirming our results Liedkte et al $2009^{(27)}$ investigated simulated external root resorption of tooth roots imaged with voxel sizes of $0.40,0.30$, and $0.20 \mathrm{~mm}$. They concluded that, even though the results from the different voxel sizes were the same, diagnosis was easier at a smaller voxel size of 0.30 or $0.20 \mathrm{~mm}$. Although the benefits of a shorter scanning time satisfy the "as low as reasonably achievable" principle, the risks of misdiagnosis and treatment complications must also be weighed. ${ }^{(30)}$

In consistence with our results Junqueira et al $2013{ }^{(11)}$ reported that CBCT is a demonstrated, effective alternative, mainly in teeth without metallic posts. Furthermore, voxel size did not significantly influence the diagnosis of vertical root fractures. Özer et al $201{ }^{(16)}$ also demonstrated that there were no significant differences regarding the diagnostic accuracy in detection of a root fracture among CBCT images of different voxel sizes. ${ }^{(32)}$

Kamburoğlu et al $2010{ }^{\text {(3) }}$ also examined the use of different voxel sizes and found that the ultraresolution Iluma images achieved better results than the low- resolution Iluma images, however, with considerably higher reconstruction times. The study showed that the dental cone-beam CT can provide useful information for the diagnosis of VRF. Not only VRF can be detected earlier using cone-beam CT compared with periapical radiography, but also fractures can be assessed in coronal, axial, and cross sectional views. ${ }^{(3,27)}$

Amintavakoli et al $2014^{\left({ }^{(14)}\right.}$ used four voxel size resolutions that showed similar results in detecting both vertical and horizontal root fractures, in vitro. However, the sensitivity of the $300 \mu \mathrm{m}$ voxel resolution was $54.0 \%$ indicating poor ability of this system in diagnosis of the presence of the both types of fractures in compared to other resolutions. The specificity reported for all four resolutions varies between $70.7 \%$ to $76.0 \%$ indicating a fair ability in diagnosis of the absence of the fractures. While Hassan et al $2010{ }^{(15)}$ in their study of comparison of five cone beam CT scan systems reported Next Generation i-CAT (with a voxel of $0.25 \mathrm{~mm}$ ) was the most accurate system followed by the Scanora $3 \mathrm{D}$ (voxel of $0.2 \mathrm{~mm}$ ), Accuitomo-XYZ (voxel of $0.25 \mathrm{~mm}$ ), Newtom $3 \mathrm{G}$ (voxel of $0.2 \mathrm{~mm}$ ), and Galileos $3 \mathrm{D}$ (voxel of $0.3 \mathrm{~mm}$ ). As well as, the ability to manipulate the images and align the image slices with possible fractures are some of the factors that may play a major role in detection of root fracture. However, these are not the only factors having a role in the detection of root fractures.

Edlund et al $2011^{(12)}$ confirmed that in addition to the superior diagnostic performance of CBCT for VRF detection that was established, the sensitivity and specificity of data with smaller voxel sizes were higher. This might indicate that a limited FOV with the smallest isotropic acquisition voxel size might enhance detection of VRF on previously endodontically treated teeth. The effect of the training and experience of the observers on the diagnostic outcome will be evaluated. Also validate CBCT for the diagnosis of VRF through establishment of its presence with surgical visualization.

In earlier studies it has been stated that axial slices were more accurate than coronal and sagittal ones for detecting vertical root fractures. However, in one clinical situation, an arc-shaped fracture line was observed in the middle third of the mesial root (of the right mandibular first molar) between post and root canal filling material in the sagittal slices, whereas no fracture line was observed in the axial 
slices. In the present study, observers were allowed to scroll axial, sagittal and coronal slices interactively to seek a fracture line in the roots, which may be recommended also in the clinical situation. ${ }^{(4,10,15)}$

Five studies compared the different voxel sizes (400 mm, $300 \mathrm{~mm}, 250 \mathrm{~mm}, 200 \mathrm{~mm}$, and 125 $\mathrm{mm}$ ) in the detection of VRFs, and the results showed that the smaller voxel sizes had a higher diagnostic accuracy. The different in vitro studies also emphasized distinct imaging aspects. Nair et al $\mathbf{2 0 0 3}^{(33)}$ compared the different image reconstruction algorithms used to diagnose VRF, and Ozer et al $\mathbf{2 0 1 0}^{(29)}$ showed that when the width of the fracture space is $400 \mathrm{~mm}$, CBCT's diagnostic accuracy is 90\%. Taramsari et al $2013^{\left({ }^{(3)}\right.}$ compared highresolution and standard zoom imaging modes and showed no significant difference between these two modes, and Melo et al $2010{ }^{(2)}$ compared four different imaging software packages, finding no significant differences between them in diagnosing VRF. Hassan et al $2010{ }^{(15)}$ showed that axial slices were more accurate than sagittal or coronal slices for detecting VRF. ${ }^{(2,3,7.15,16,29)}$

The diagnostic accuracy of VRF in both in vivo and in vitro studies is different among these studies. One in vitro study showed a diagnostic accuracy of as high as $100 \%$. However, the highest reported diagnostic accuracy in the human studies was only 93\% from a sample size of 14 patients. Therefore, the results of the in vitro studies might not reflect the real clinical efficacy in detecting VRFs. ${ }^{(7,13)}$

Patel et al 2014 ${ }^{(35)}$ observed that for larger lesions, no significant difference was noted between $0.2-\mathrm{mm}$ and $0.4-\mathrm{mm}$ voxel sizes, whereas for smaller lesions, the lower resolution protocol (0.4-mm voxel size) rendered a significantly lower sensitivity value (67.3\%). ${ }^{(21)}$

\section{CONCLUSION}

The CBCT provides enhanced and accurate information for the diagnosis of root fractures, thereby constituting an excellent alternative for diagnosis in the dental practice. However, clinical signs and symptoms are fundamental and very important for the diagnosis of fractures, besides auxiliary resources as the CBCT.

We think that not only the voxel size exerts little influence on the detection of root fractures but also the accuracy levels were lower when using the voxels of greater thickness, so, we recommend using the smallest voxel size but if it is not available shift to the higher size as it will not affect the clinical outcome to any significant degree.

\section{REFERENCES}

1- Youssefzadeh S, Gahleitner A, Dorffner R, Bernhart T and Kainberger FM: Dental vertical root fractures: value of CT in detection. Radiology 1999; 210: 545-9.

2- Melo SLS, Bortoluzzi EA, Abreu M Jr, et al: Diagnostic ability of a cone-beam computed tomography scan to assess longitudinal root fractures in prosthetically treated teeth. J Endod 2010; 36: 1879-82.

3- Kamburoğlu K, Murat S, Yüksel SP, et al: Detection of vertical root fracture using cone-beam computerized tomography: an in vitro assessment. Oral Surg Oral Med Oral Pathol Oral Radiol Endod 2010; 109: e74-81.

4- Zou X, Liu D, Yue L and Wu M: The ability of cone-beam computerized tomography to detect vertical root fractures in endodontically treated and nonendodontically treated teeth: a report of 3 cases. Oral Surg Oral Med Oral Pathol Oral Radiol Endod 2011; 111: 797-801.

5- Bechara B, McMahan C, Nasseh I, Geha H, Hayek E, Khawam G, Raad M and Noujeim M: Number of basis images effect on detection of root fractures in endodontically treated teeth using a cone beam computed tomography machine: an in vitro study. Oral Surg Oral Med Oral Pathol Oral Radiol 2013; 115: 676-681.

6- Corbella S, Del Fabbro M, Tamse A, Rosen E, Tsesis I and Taschieri S: Cone beam computed tomography for the diagnosis of vertical root fractures: a systematic review of the literature and meta-analysis. Oral Surg Oral Med Oral Pathol Oral Radiol 2014; 118: 593-602.

7- Huang C and Lee B: Diagnosis of vertical root fracture in endodontically treated teeth using computed tomography $\mathrm{J}$ Dent Sci. 2015; 1-6. Article in press. 
8- Bernardes R, de Moraes I, Duarte M, Azevedo B, de Azevedo J and Bramante C: Use of cone-beam volumetric tomography in the diagnosis of root fractures. Oral Surg Oral Med Oral Pathol Oral Radiol Endod 2009; 108: 270-277.

9- Hassan B, Metska ME, Ozok AR, et al: Detection of vertical root fractures in endodontically treated teeth by cone beam computed tomography. J Endod 2009; 35: 719-22.

10- Ferreira R, Bahrami G, Isidor F, Wenzel A, Haiter-Neto F and Groppo F: Detection of vertical root fractures by conebeam computerized tomography in endodontically treated teeth with fiber-resin and titanium posts: an in vitro study. Oral Surg Oral Med Oral Pathol Oral Radiol 2013; 115: e49-e57.

11- Junqueira R, Verner F, Campos C, Devito K and do Carmo A: Detection of vertical root fractures in the presence of intracanal metallic post: A comparison between periapical radiography and cone-beam computed tomography. J Endod 2013; 39: 1620-1624.

12- Edlund M, Nair M and Nair U: Detection of vertical root fractures by using cone-beam computed tomography: A clinical study. J Endod 2011; 37: 768-772.

13- Metska M, Aartman I, Wesselink P and Özok A: Detection of vertical root fractures in vivo in endodontically treated teeth by cone-beam computed tomography scans. J Endod 2012; 38: 1344-1347.

14- Amintavakoli N, Pharoah MJ, Basrani B and Lam EW: The effect of voxel size on the identification of vertical and horizontal root fractures by cone beam computed tomography: an in vitro study. Oral Surg Oral Med Oral Pathol Oral Radiol, 2014; 117: Page e403.

15- Hassan B, Metska ME, Ozok AR, et al: Comparison of five cone-beam computed tomography systems for the detection of vertical root fractures. J Endod 2010; 36: 126-9.

16- Özer SY: Detection of vertical root fractures by using cone beam computed tomography with variable voxel sizes in an in vitro model. J Endod 2011; 37: 75-9.

17- Sirin Y, Guven K, Horasan S, Sencan S, Bakir B, Barut O, Tanyel C, Aral A and Firat D: The influence of secondary reconstruction slice thickness on NewTom $3 \mathrm{G}$ cone beam computed tomography-based radiological interpretation of sheep mandibular condyle fractures. Oral Surg Oral Med Oral Pathol Oral Radiol Endod 2010; 110: 638-647.

18- de-Azevedo-Vaz S, de Faria Vasconcelos K, Neves F, Melo S, Campos P and Haiter-Neto F: Detection of periimplant fenestration and dehiscence with the use of two scan modes and the smallest voxel sizes of a cone-beam computed tomography device. Oral Surg, Oral Med, Oral Pathol, Oral Radiol 2013; 115: 121-127.

19- Hashem D, Brown J, Patel S, Mannocci F, Donaldson $\mathrm{N}$, Watson $\mathrm{T}$ and Banerjee A: An in vitro comparison of the accuracy of measurements obtained from high- and low-resolution cone-beam computed tomography scans. J Endod 2013; 39: 394-397.

20- Ye N, Long H, Xue J, Wang S, Yang X and Lai W: Integration accuracy of laser-scanned dental models into maxillofacial cone beam computed tomography images of different voxel sizes with different segmentation threshold settings. Oral Surg Oral Med Oral Pathol Oral Radiol 2014; 117: 780-786.

21- Yadav S, Palo L, Mahdian M, Upadhyay M and Tadinadad A: Diagnostic accuracy of 2 cone-beam computed tomography protocols for detecting arthritic changes in temporomandibular joints. Am Journal of Orthod Dentofacial Orthop 2015; 147: 339-44.

22- Korostoff J, Aratsu A, Kasten B and Mupparapu M: Radiologic Assessment of the Periodontal Patient. Dent Clin N Am 2016; 60: 91-104.

23- Sinanoglu A, Demirturk Kocasarac H and Noujeim M: Age estimation by an analysis of spheno-occipital synchondrosis using cone-beam computed tomography. Leg Med 2016; 18: 13-19.

24- Lukat T, Perschbacher S, Pharoah M and Lam E: The effects of voxel size on cone beam computed tomography images of the temporomandibular joints. Oral Surg Oral Med Oral Pathol Oral Radiol 2015; 119: 229-237.

25- Wenzel A, Haiter-Neto F, Frydenberg M, et al: Variable-resolution cone-beam computerized tomography with enhancement filtration compared with intraoral photostimulable phosphor radiography in detection of transverse root fractures in an in vitro model. Oral Surg Oral Med Oral Pathol Oral Radiol Endod 2009; 108: 939-45.

26- Librizzi Z, Tadinada A, Valiyaparambil J, Lurie A and Mallya S: Cone-beam computed tomography to detect erosions of the temporomandibular joint: Effect of field of view and voxel size on diagnostic efficacy and effective dose. Am J Orthod Dentofacial Orthop 2011; 140: e25-e30.

27- Liedke GS, Dias da Silviera HE, Dias da Silviera HL, Duntra V and Poli de Figueiredo A: Influence of voxel size in the diagnostic ability of cone beam tomography to evaluate simulated external root resorption. J Endod 2009; 35: 233-5. 
28- Maloul A, Fialkov J and Whyne C: The impact of voxel size-based in accuracies on the mechanical behavior of thin bone structures. Ann Biomed Eng. 2011; 39:1092-1100.

29- Özer SY: Detection of vertical root fractures of different thicknesses in endodontically enlarged teeth by cone beam computed tomography versus digital radiography. J Endod 2010; 36: 1245-9.

30- Damstra J, Fourie Z, Slater J and Renc Y: Accuracy of linear measurements from cone-beam computed tomographyderived surface models of different voxel sizes. Am J Orthod Dentofacial Orthop 2010; 137: 16.e1-e6.

31- Ballrick JW, Palomo JM, Ruch E, Amberman BD and Hans MG: Image distortion and spatial resolution of a commercially available cone-beam computed tomography machine. Am J Orthod Dentofacial Orthop 2008; 134:573-82.

32- Choi J, Lee S, Choi S, Heo M, Huh K, Yi W, Kang S, Han D and Kim E: Relationship between physical factors and subjective image quality of cone-beam computed tomography images according to diagnostic task. Oral Surg Oral Med Oral Pathol Oral Radiol 2015; 119:357-365.

33- Nair MK, Grondahl HG, Webber RL, Nair UP and Wallace JA: Effect of iterative restoration on the detection of artificially induced vertical radicular fractures by tuned aperture computed tomography. Oral Surg Oral Med Oral Pathol Oral Radiol Endod 2003; 96: 118-25.

34- Taramsari M, Kajan ZD, Bashirzadeh P and Salamat F: Comparison of high-resolution and standard zoom imaging modes in cone beam computed tomography for detection of longitudinal root fracture: an in vitro study. Imaging Sci Dent 2013; 43: 171-7.

35- Patel A, Tee BC, Fields H, Jones E, Chaudhry J and Sun $\mathrm{Z}$ : Evaluation of cone-beam computed tomography in the diagnosis of simulated small osseous defects in the mandibular condyle. Am J Orthod Dentofacial Orthop 2014; 145: 143-56. 